\title{
Studying Evolution with Jets at STAR
}

\author{
Renee Fatemi* \\ University of Kentucky E-mail: renee. fatemi@uky • edu
}

For over a decade the Relativistic Heavy Ion Collider (RHIC) at Brookhaven National Laboratory has facilitated the development of a rich and diverse proton structure program by consistently providing highly polarized proton collisions over a wide range of center-of-mass energies. The STAR collaboration has capitalized on the versatility of the RHIC beams and its own wide acceptance detector to build a multi-faceted jet program. This contribution will discuss STAR's recent mid-rapidity jet measurements and their sensitivity to the gluon momentum, gluon helicity and quark transversity distributions.

QCD Evolution 2015 -QCDEV2015-

26-30 mAY 2015

Jefferson Lab (JLAB), Newport News Virginia, USA

${ }^{*}$ Speaker. 


\section{Jet Reconstruction with the STAR detector at RHIC}

The Relativistic Heavy Ion Collider (RHIC) located at Brookhaven National Laboratory provides an excellent environment to study evolution effects in both spin-averaged and spin-dependent reactions. RHIC is an extremely versatile machine, capable of providing collisions of longitudinal and transversely protons at center-of-mass energies up to $\sqrt{s}=510 \mathrm{GeV}$. The Solenoidal Tracker at RHIC (STAR) [1] is a large acceptance detector equipped with a time projection chamber (TPC) for track trajectory and momentum reconstruction, as well as particle identification for charged particles scattered at mid-rapidity $(|\eta|<1.4)$. Electromagnetic calorimeters (EMC) provide triggering capabilities as well as electromagnetic energy and position information for $-1<\eta<2$. STAR's wide detector coverage in rapidity and $2 \pi$ coverage in $\phi$ facilitates full jet reconstruction at mid-rapidity. Several sub-systems, the Vertex Position Detector (VPD), Zero Degree Calorimeter (ZDC) and Beam Beam Counter (BBC), provide luminosity and local polarimetry measurements.

The ability to fully reconstruct jets with transverse momentum $p_{T}$ ranging from $5-120 \mathrm{GeV}$, at a variety of center-of-mass energies, allows STAR to track how jet observables change for an average partonic momentum fraction $\langle x\rangle$ as a function of momentum transfer $Q$. Jets are especially useful tools for evolution studies as they provide access to the scattered parton momentum as well as information about fragmentation effects within the jet. This proceeding will discuss STAR's ability to utilize jets to study evolution effects in the gluon momentum and helicity distributions as well as the quark transversity distributions.

\section{Constraining High-x Gluon Momentum Distributions}

The unpolarized parton distribution functions (PDFs), denoted as $f\left(x, Q^{2}\right)$, describe the number density of partons inside the proton with flavor $f$ and carrying longitudinal momentum fraction $x$. These distributions [2] are extracted from global analyses that incorporate experimental data from lepton-hadron and hadron-hadron collisions into a theoretical perturbative QCD framework. A recent analysis by the CTEQ-TEA collaboration [3] demonstrates that the large and rapidly increasing uncertainty for gluons, $g\left(x, Q^{2}\right)$, in the $x>0.3$ regime is driven by the uncertainties in the existing data. The jets reconstructed at moderately high transverse momentum, $p_{T}>30 \mathrm{GeV}$, in $\sqrt{s}=200 \mathrm{GeV}$ collisions at STAR are sensitive to gluons in this regime and may be able to provide new constraints on $g\left(x, Q^{2}\right)$.

Figure 1 shows the mid-rapidity inclusive jet cross-section from $19 p b^{-1}$ of $\sqrt{s}=200 \mathrm{GeV} p+p$ collisions. Jets are reconstructed from TPC tracks and EMC towers using an anti- $k_{T}$ jet algorithm [4] [5] with radius parameter $R=0.6$. The detected jet spectrum is unfolded to the particle level using simulations generated from PYTHIA 6.426 [6] tuned to Perugia 0 [7] for the $p+p$ collisions and utilizing GEANT 3 for the detector emulation. The systematic errors on the corrected jet yields include contributions from tracking and calorimeter resolution and inefficiencies, but the dominant source of uncertainty arises from the electromagnetic calorimeter calibration. 


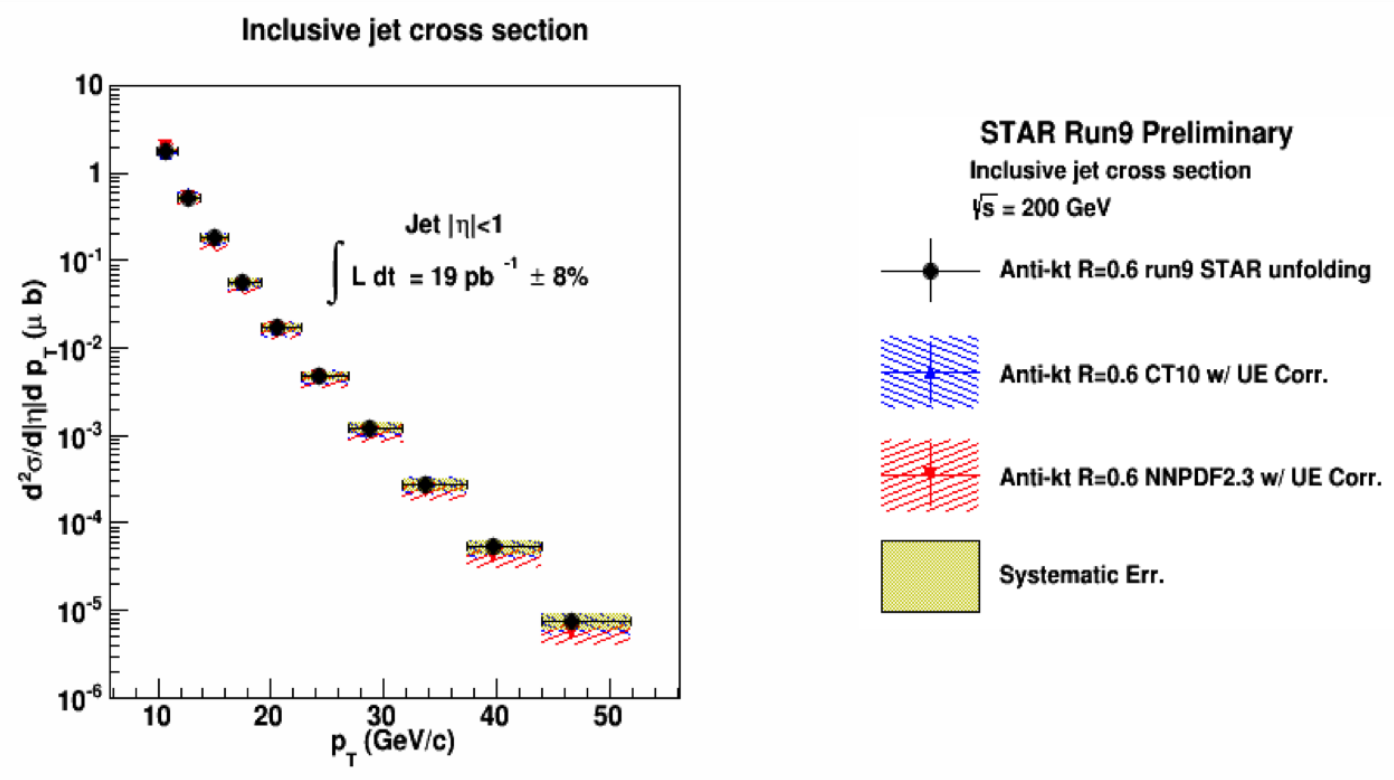

Figure 1: The inclusive jet cross section in $\sqrt{s}=200 \mathrm{GeV}$ for jets with $|\eta|<1.0$. The results are compared with NLO pQCD calculations that incorporate underlying event and hadronization corrections for the CT10 (blue) [8] and NNPDF3.0 (red) [9] PDF sets.

Due to hadronization effects and underlying event contributions, jet yields corrected to the particle level cannot be directly compared with next-to-leading order (NLO) perturbative QCD (pQCD) calculations. Hadronization and underlying event contributions are extracted through comparisons of the jet cross-section at the particle and parton level for each jet $p_{T}$ bin. Parton jets consist of the partons that participated in the hard collision as well as fragmentation partons originating from initial state and final state radiation. Partons arising from the underlying event and interactions with the proton remnants are excluded. Clearly the nature of these parton jets depends on the fragmentation model implemented in the generator and are likely to change with shower model and tune parameters. In an effort to separate these effects from experimental systematic errors the hadronization and underlying event correction, which does not exceed 5\%, is applied to selected NLO pQCD calculations. The data are in good agreement within the scaling uncertainties of both the CT10[8] and NNPDF3.0[9] calculations represented by blue and red hashed blocks respectively. Additional plots and analysis details on the 2009 inclusive jet cross-section may be found in [10].

\section{Constraining Low-x Gluon Helicity Distributions}

The helicity distributions, $\Delta f\left(x, Q^{2}\right)$, describe the number density of longitudinally polarized partons with flavor $f$ inside a longitudinally polarized proton. Traditionally these distributions are accessed via double spin asymmetry measurements $\left(A_{L L}\right)$, defined as the difference of yields with the beam and target spins aligned versus anti-aligned, normalized by the sum. Recent global analyses [12,13] illustrate the relatively strong constraints on the up and down quark helicity PDFs provided by decades of fixed target polarized lepton-proton scattering experiments. These same experiments are not sensitive to $\Delta g\left(x, Q^{2}\right)$ at leading order and provide only limited constraints on 
the polarized gluon distribution.

Collisions of high energy longitudinally polarized protons at RHIC provide direct access to quarkgluon and gluon-gluon scattering, opening a unique opportunity to map out $\Delta g\left(x, Q^{2}\right)$ over a wide range of momentum transfers. Initial inclusive jet $[19,20,21]$ and pion $[22,23,24,25] A_{L L}$ measurements from $\sqrt{s}=200 \mathrm{GeV} \mathrm{p}+\mathrm{p}$ collisions at RHIC established that the gluon helicity distribution in the region $0.05<x<0.2$ is not large. Only recently have these measurements attained the precision needed to clearly show that the gluons in this kinematic range are positively polarized. This is demonstrated in figure 2 which shows STAR's recently published inclusive jet $A_{L L}$ [26] measured from $20 \mathrm{pb}^{-1}$ of $\sqrt{s}=200 \mathrm{GeV} \mathrm{p}+\mathrm{p}$ collisions collected in 2009. The data points are systematically larger than the predictions incorporating the 2008 DSSV fits [11, 12], which determined the gluon helicity PDFs to be consistent with zero at a scale of $Q^{2}=10 \mathrm{GeV}^{2}$.

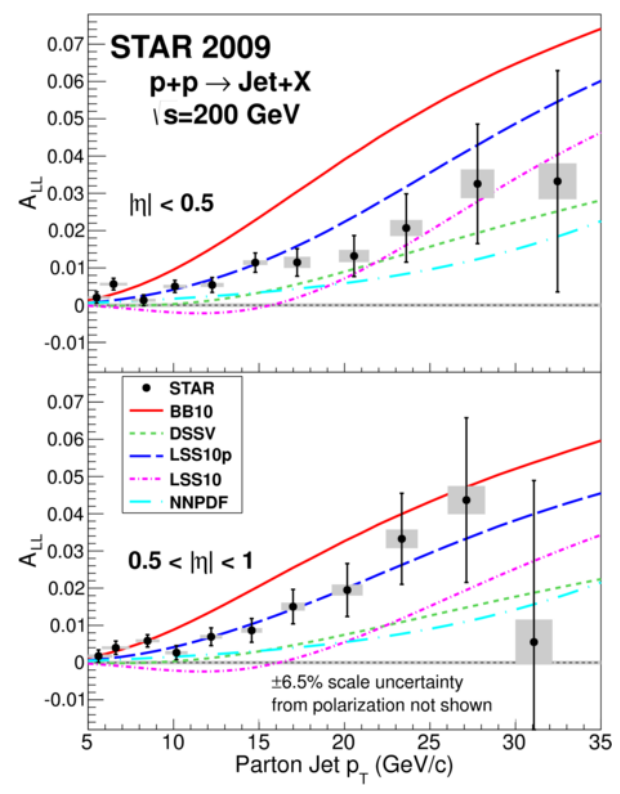

Figure 2: The 2009 STAR inclusive jet $A_{L L}$ data [26] plotted with NLO pQCD predictions for a variety PDF sets $[11,12,14,15,16]$.

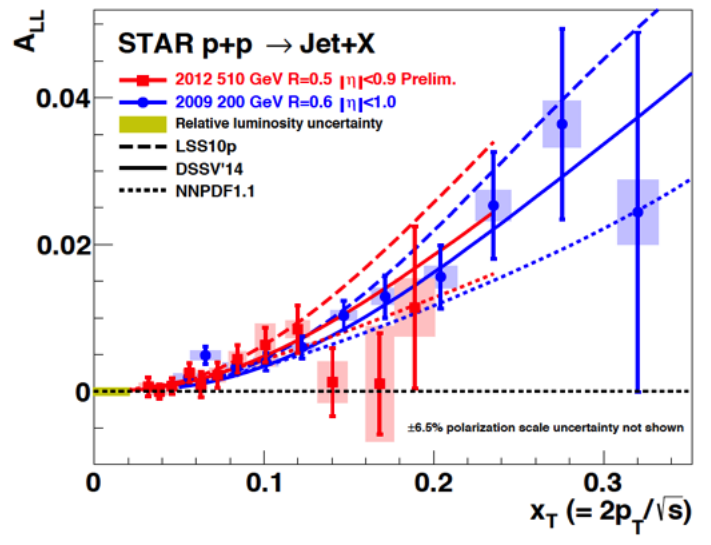

Figure 3: The red (blue) points are the STAR inclusive jet $A_{L L}$ for mid-rapidity jets reconstructed from $\sqrt{s}=510(\sqrt{s}=200) \mathrm{GeV} \mathrm{p}+\mathrm{p}$ collisions collected in 2012 (2009). The curves are NLO pQCD theory predictions using the LSS10p [15], DSSV'14 [18] and NNPDF1.1 [13] PDF sets. STAR's 2009 inclusive jet $A_{L L}$ data are included in the DSSV'14 and NNPDF1.1 curves.

As in the cross-section analysis, the jets in the 2009 asymmetry analysis were reconstructed with an anti-kT jet algorithm and cone parameter $\mathrm{R}=0.6$. Unlike the cross-section analysis, these jets were corrected back to the parton level in order to facilitate the most direct comparison to the NLO pQCD curves plotted for comparison on figure 2. The theoretical curves were generated by inserting the corresponding polarized PDFs $[11,12,14,15,16]$ into Mukherjee and Vogelsang's NLO jet production code [17]. The DSSV [18] and NNPDF [13] collaborations included STAR's 2009 inclusive jet results into their fits and extracted consistent values for the total gluon contribution to the proton spin at $Q^{2}=10 \mathrm{GeV}^{2}, \int_{0.05}^{1.0} \Delta g(x) d x=0.2_{-0.07}^{+0.06}$ and $\int_{0.05}^{0.2} \Delta g(x) d x=0.17 \pm 0.06$ respectively.

While these measurements represent a leap forward in our understanding of the gluon helicity distribution, they provide very limited information about $\Delta g\left(x, Q^{2}\right)$ below $x \sim 0.05$. This lack of data 
translates directly into a large uncertainty on the total gluon contribution to the spin of the proton $\Delta G=\int_{0}^{1} \Delta g(x)$. One way to gain sensitivity to lower $x$ regimes is to repeat the analysis at higher center-of-mass energies. In 2012 STAR collided longitudinal polarized protons with an average of $53 \%$ polarization at $\sqrt{s}=510 \mathrm{GeV}$. The black points in figure 3 represent the mid-rapidity inclusive jet $A_{L L}$ from the 2012 dataset. This analysis followed the 2009 analysis closely, except for the cone parameter $\mathrm{R}=0.5$, which was implemented to reduce effects from the underlying event and pile-up contributions that increase at higher center of mass energies. The 2012 result is plotted as a function of $x_{T}=2 p_{T} / \sqrt{s}$ to facilitate a comparison with the 2009 mid-rapidity $A_{L L}$ shown in red. The results agree well in the region of overlap and the extension to lower $x_{T}$ for the $\sqrt{s}=510 \mathrm{GeV}$ is clear. Additional analysis details on the 2012 inclusive jet $A_{L L}$ may be found in [27].

\section{Accessing Quark Transversity Distributions for the first time in pp Collisions}

The transversity distribution, $\Delta_{T} f\left(x, Q^{2}\right)$, describes the number density of transversely polarized partons with flavor $f$ inside a transversely polarized proton. Although all three PDFs, momentum, helicity and transversity, are essential to completely describe the structure of the proton, we know comparatively little about the transversity distributions. Recent global analyses [28, 29] indicate that up quark transversity is positive and maximal in the valence region, while down quark transversity is smaller and likely negative, although the size of the error envelope makes the sign uncertain. Unlike the momentum and helicity distributions transversity is chiral odd and can be observed only when coupled to another chiral-odd distribution. Although there are several possible chiral-odd distributions that could be coupled with $\Delta_{T} f\left(x, Q^{2}\right)$ this proceedings will focus on the Collins and Interference fragmentation function distributions.

The Collins fragmentation function $H_{1}^{\perp}\left(z, j_{T}\right)$ describes the probability for a transversely polarized quark to fragment into a hadron that carries momentum fraction $z=p^{\text {hadron }} / p^{\text {quark }}$ and transverse momentum $j_{T}$ with respect to $p^{\text {quark }}$. Because $H_{1}^{\perp}$ is dependent on $j_{T}$ it is defined within the transverse momentum dependent (TMD) framework. The Interference fragmentation function (IFF) describes the fragmentation of a transversely polarized quark into a di-hadron pair. In contrast to the Collins FF, the IFF survives integration over the $j_{T}$ of the hadrons and is defined within the traditional collinear framework. Previously these distributions have been accessed only in semiinclusive deep inelastic lepton-nucleon scattering [30, 31, 32, 33, 34] and e+e- [35, 36] scattering experiments, inviting questions of how they change when measured in hadronic collisions and how they evolve with momentum transfer scales.

In hadronic collisions the single spin asymmetry of the azimuthal distribution of charged pions within a jet, $A_{U T}^{\sin \left(\phi_{S}-\phi_{H}\right)}$, provides sensitivity to the convolution of quark transversity and Collins FF distributions, $\Delta_{T} f\left(x, Q^{2}\right) \otimes H_{1}^{\perp}\left(z, j_{T}\right)$. Here $\phi_{S}$ and $\phi_{H}$ define the angles between the reaction plane and the proton spin and the reaction plane and the $j_{T}$ vector respectively. In 2011 (2012) STAR collected $25(22) \mathrm{pb}^{-1}$ of 53\% (63\%) transversely polarized proton collisions at $\sqrt{s}=500$ (200) $\mathrm{GeV}$. In both datasets jets were reconstructed using the anti- $k_{T}$ algorithm, with a cone parameter of $\mathrm{R}=0.5$ and 0.6 for $\sqrt{s}=500$ and $200 \mathrm{GeV}$ respectively. Figure 4 shows the Collins $A_{U T}$ as a function of measured $z=p_{\text {pion }} / p_{\text {jet }}$ for $\sqrt{s}=200$ (blue) and 500 (red) GeV $p^{\uparrow}+p$ collisions. 


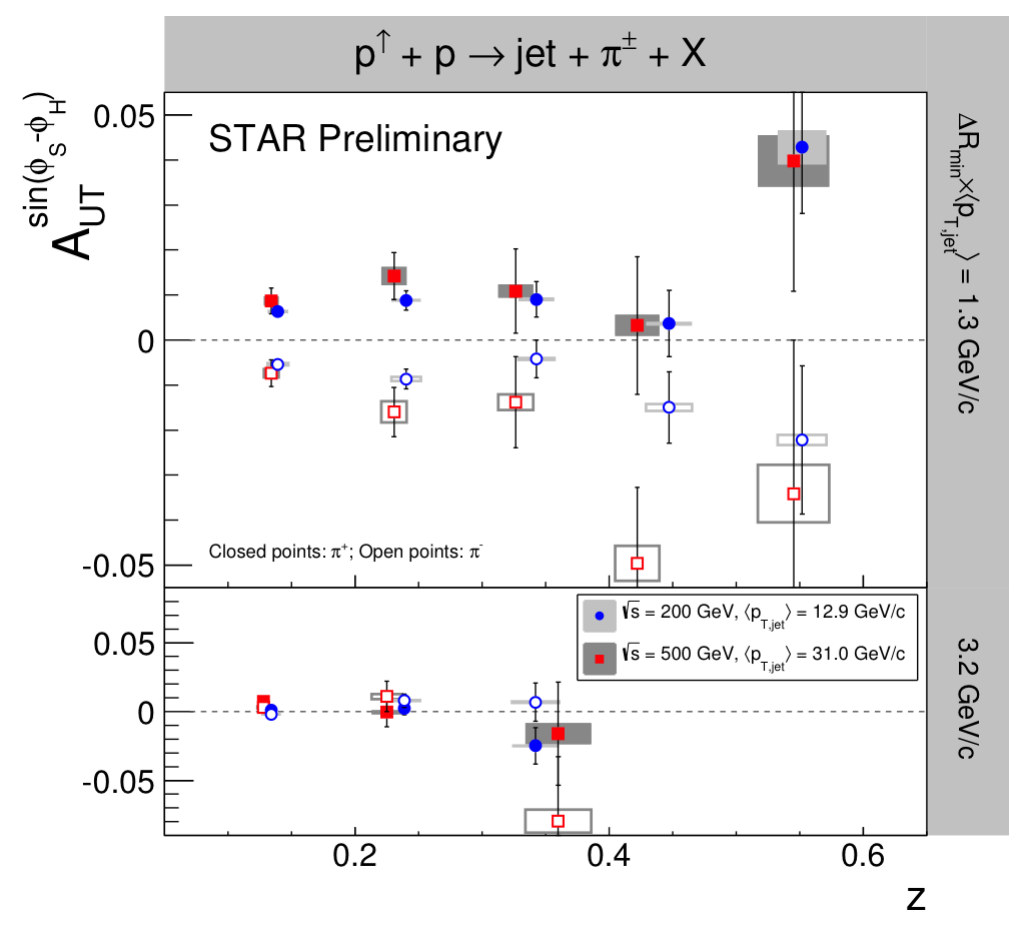

Figure 4: STAR Collins $A_{U T}$ as a function of $z$ for positively (closed) and negatively (open) charged pions in jets reconstructed from $\sqrt{s}=200$ (blue) and 500 (red) $\mathrm{GeV} p^{\uparrow}+p$ collisions.

Both sets of asymmetries select on forward scattering jets $\left.\left(\eta^{\text {jet }}>\right) 0\right)$ with respect to the polarized beam. The jet $p_{T}$ bins for each beam energy have been chosen to sample approximately the same quark $x$ values. The angular cuts, characterized by the minimum distance of the charged pion from the jet thrust axis, have been chosen to sample the same $j_{T}$ values $\left(\sim \Delta R \times z \times p_{T}^{\text {jet }}\right)$. The fact the asymmetries change with average $j_{T}$ indicates a significant $j_{T}$ dependence. The evolution of the Collins asymmetries from $\sqrt{s}=200$ to $500 \mathrm{GeV}$ is consistent with $x_{T}$ scaling. Additional details on the 2011 and 2012 Collins $A_{U T}$ analyses may be found in [38, 37].

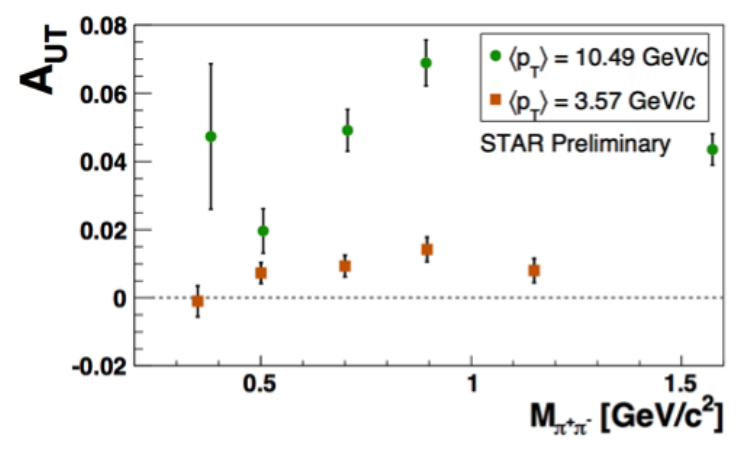

Figure 5: STAR IFF asymmetries for $\pi^{+} \pi^{-}$in $\sqrt{s}=200 \mathrm{GeV} p^{\uparrow}+p$ collisions.
In the same datasets used for the Collins analysis, STAR has measured the $\pi^{+} \pi^{-}$correlation single spin asymmetry $A_{U T}^{\sin \left(\phi_{R}-\phi_{S}\right)}$, which is sensitive to the convolution of the quark transversity and IFF distributions, $\Delta_{T} f\left(x, Q^{2}\right) \otimes H_{1}^{\varangle}\left(z, j_{T}\right)$. In an effort to restrict correlations to those pions that fragment from the same parent quark a $\Delta R=$ $\sqrt{\Delta \eta^{2}+\Delta \phi^{2}}<0.7$ requirement is placed on the distance in $\eta-\phi$ space between the two pions. The reaction plane is defined by the incoming protons beams and the sum of the three momentum of the two pions $\left(\overrightarrow{p_{H}}\right)$. Accordingly 
$\phi_{S}$ and $\phi_{R}$ define the angle between the reaction plane and the proton spin and the reaction plane and the vector difference between the two pion momentums respectively.



Figure 6: STAR IFF asymmetries for $\pi^{+} \pi^{-}$in $\sqrt{s}=500 \mathrm{GeV} p^{\uparrow}+p$ collisions.

Figures 5 and 6 plot the IFF asymmetry for $\eta^{\pi+\pi-}>0$ as a function of the invariant mass $M_{i n v}^{\pi+\pi-}$ of the $\pi^{+} \pi^{-}$pair for $\sqrt{s}=200$ and $\sqrt{s}=500 \mathrm{GeV}$. Data is only shown for a low and high $p_{T}^{\pi^{+}} \pi^{-}$bin, although additional bins were calculated. As expected $A_{U T}^{\sin \phi_{R}-\phi_{S}}$ increases with $p_{T}^{\pi^{+}} \pi^{-}$. The highest bin shows a possible enhancement around the $\rho$ meson mass, an expected effect if the IFF asymmetry is due to the interference of a vector meson decay in a relative p-wave interfering with a non-resonance background in a relative s-wave. Additional analysis details on the 2006, 2011 and 2012 IFF $A_{U T}$ analyses may be found in $[39,40]$.

\section{Conclusions}

Proton structure studies are an essential component of the nuclear physics community's long standing mission to understand the nature of quark and gluon interactions. Hadron colliders like RHIC play a vital role in these studies by providing transversely and longitudinally polarized proton collisions over a wide range of center of mass energies, allowing experimentalists to vary the scale of the partonic interactions and therefore examine evolution effects within QCD. Hadronic collisions also provide direct access to gluon interactions making them a natural place to study gluon distributions within the proton. STAR's ability to measure high $p_{T}$ inclusive jet cross-sections in $\sqrt{s}=200 \mathrm{GeV}$ collisions opens the window to new high $x$ constraints on $g\left(x, Q^{2}\right)$. Measurements of the spin dependence of these same jets provided the first evidence of polarized gluons in the valence region. By reconstructing jets at relatively low $p_{T}$ in $\sqrt{s}=510 \mathrm{GeV}$ collisions, STAR has pushed the sensitivity of these asymmetry measurements to lower $x$, where large uncertainties on $\Delta g\left(x, Q^{2}\right)$ remain.

STAR's ability to simultaneously measure the IFF and Collins asymmetries provides a unique opportunity to study the universality and evolution of the transversity distributions, interference and Collins fragmentation functions. The fact that these asymmetries are measured in the same system, at both $\sqrt{s}=200$ and $500 \mathrm{GeV}$, will help untangle universality and evolution effects. These asymmetries may also provide insights into the size and nature of factorization breaking effects that are expected to affect the Collins asymmetries in hadronic collisions. In the IFF channel the transversity distributions and fragmentation functions are collinear, for which factorization is in general better understood. The Collins channel accesses the same physics from the same events, only formulated with the TMD framework needed to describe the Collins functions. This duality will allow theorists to further study and develop the TMD framework within hadronic collisions. 


\section{References}

[1] K. H. Ackermann et al. [STAR Collaboration], Nucl. Instrum. Meth. A 499, 624 (2003).

[2] A. Buckley, J. Ferrando, S. Lloyd, K. NordstrŽm, B. Page, M. R§fenacht, M. SchŽnherr and G. Watt, Eur. Phys. J. C 75, 132 (2015)

[3] J. Gao et al., Phys. Rev. D 89, no. 3, 033009 (2014)

[4] M. Cacciari, G. P. Salam and G. Soyez, JHEP 0804, 063 (2008)

[5] M. Cacciari, G. P. Salam and G. Soyez, Eur. Phys. J. C 72 (2012) 1896

[6] T. Sjostrand, S. Mrenna and P. Z. Skands, JHEP 0605, 026 (2006)

[7] P. Z. Skands, arXiv:0905.3418 [hep-ph].

[8] H. L. Lai, M. Guzzi, J. Huston, Z. Li, P. M. Nadolsky, J. Pumplin and C.-P. Yuan, Phys. Rev. D 82, 074024 (2010)

[9] R. D. Ball et al. [NNPDF Collaboration], JHEP 1504, 040 (2015)

[10] X. Li [STAR Collaboration], in Proceedings of the 23rd International Workshop on Deep-Inelastic Scattering and Related Subjects (DIS 2015) (2015).

[11] D. de Florian, R. Sassot, M. Stratmann and W. Vogelsang, Phys. Rev. Lett. 101, 072001 (2008)

[12] D. de Florian, R. Sassot, M. Stratmann and W. Vogelsang, Phys. Rev. D 80, 034030 (2009)

[13] E. R. Nocera et al. [NNPDF Collaboration], Nucl. Phys. B 887, 276 (2014)

[14] J. Blumlein and H. Bottcher, Nucl. Phys. B 841, 205 (2010)

[15] E. Leader, A. V. Sidorov and D. B. Stamenov, Phys. Rev. D 82, 114018 (2010)

[16] R. D. Ball et al. [NNPDF Collaboration], Nucl. Phys. B 874, 36 (2013)

[17] A. Mukherjee and W. Vogelsang, Phys. Rev. D 86, 094009 (2012)

[18] D. de Florian, R. Sassot, M. Stratmann and W. Vogelsang, Phys. Rev. Lett. 113, no. 1, 012001 (2014)

[19] B. I. Abelev et al. [STAR Collaboration], Phys. Rev. Lett. 97, 252001 (2006)

[20] B. I. Abelev et al. [STAR Collaboration], Phys. Rev. Lett. 100, 232003 (2008)

[21] L. Adamczyk et al. [STAR Collaboration], Phys. Rev. D 86, 032006 (2012)

[22] S. S. Adler et al. [PHENIX Collaboration], Phys. Rev. D 73, 091102 (2006)

[23] A. Adare et al. [PHENIX Collaboration], Phys. Rev. D 76, 051106 (2007)

[24] A. Adare et al. [PHENIX Collaboration], Phys. Rev. D 79, 012003 (2009)

[25] A. Adare et al. [PHENIX Collaboration], Phys. Rev. D 90, no. 1, 012007 (2014)

[26] L. Adamczyk et al. [STAR Collaboration], Phys. Rev. Lett. 115, no. 9, 092002 (2015)

[27] Z. Chang [STAR Collaboration], in Proceedings of the 21st International Symposium on Spin Physics (SPIN 2015) (2015).

[28] Z. B. Kang, A. Prokudin, P. Sun and F. Yuan, arXiv:1505.05589 [hep-ph]

[29] M. Anselmino, M. Boglione, U. DÕAlesio, J. O. Gonzalez Hernandez, S. Melis, F. Murgia and A. Prokudin, Phys. Rev. D 92, no. 11, 114023 (2015) 
[30] A. Airapetian et al. [HERMES Collaboration], Phys. Rev. Lett. 94, 012002 (2005)

[31] A. Airapetian et al. [HERMES Collaboration], JHEP 0806, 066 (2008)

[32] C. Adolph et al. [COMPASS Collaboration], Phys. Lett. B 713, 10 (2012)

[33] M. G. Alekseev et al. [COMPASS Collaboration], Phys. Lett. B 692, 240 (2010)

[34] M. Alekseev et al. [COMPASS Collaboration], Phys. Lett. B 673, 127 (2009)

[35] R. Seidl et al. [Belle Collaboration], Phys. Rev. D 78, 032011 (2008) [Phys. Rev. D 86, 039905 (2012)]

[36] A. Vossen et al. [Belle Collaboration], Phys. Rev. Lett. 107, 072004 (2011)

[37] J. L. Drachenberg [STAR Collaboration], in Proceedings of the 2 3rd International Workshop on Deep-Inelastic Scattering and Related Subjects (DIS 2015) (2015).

[38] J. K. Adkins and J. L. Drachenberg [STAR Collaboration], in Proceedings of the 21st International Symposium on Spin Physics (SPIN 2015) (2015).

[39] L. Adamczyk et al. [STAR Collaboration], Phys. Rev. Lett. 115, 242501 (2015)

[40] M. Skoby [STAR Collaboration], in Proceedings of the 21st International Symposium on Spin Physics (Spin 2014) (2015) 